\title{
Le mobilier du « dolmen de Saint-Père-en-Retz » (Loire-Atlantique) : nouveaux éléments de réflexion
}

The finds of the "dolmen de Saint-Père-en-Retz" (Loire-Atlantique): new evidence

El material del "dolmen de Saint-Père-en-Retz" (Loire-Atlantique): nuevos elementos de reflexión

Das Fundinventar aus dem "Dolmen von Saint-Père-en-Retz" (Loire-Atlantique, Pays-de-Loire, France) : neue Forschungserkenntnisse

Henri Gandois et Laure Barthet

\section{(2) OpenEdition}

\section{Journals}

Édition électronique

URL : https://journals.openedition.org/rao/2914

DOI : $10.4000 /$ rao. 2914

ISBN : 978-2-7535-5014-8

ISSN : $1775-3732$

Éditeur

Presses universitaires de Rennes

Édition imprimée

Date de publication : 31 décembre 2015

Pagination : 83-95

ISBN : $978-2-7535-5012-4$

ISSN : 0767-709X

\section{Référence électronique}

Henri Gandois et Laure Barthet, « Le mobilier du « dolmen de Saint-Père-en-Retz » (Loire-Atlantique) : nouveaux éléments de réflexion », Revue archéologique de l'Ouest [En ligne], 32 | 2015, mis en ligne le 28 avril 2018, consulté le 23 août 2022. URL : http://journals.openedition.org/rao/2914 ; DOI : https:// doi.org/10.4000/rao.2914 


\title{
Le mobilier du « dolmen de Saint-Père-en-Retz » (Loire-Atlantique) : nouveaux éléments de réflexion
}

\author{
The Finds of the "dolmen de Saint-Père-en-Retz" (Loire-Atlantique): New Evidence
}

\author{
Henri GANDOIS ${ }^{a}$ et Laure BARTHET ${ }^{b}$
}

\begin{abstract}
Résumé : L'étude des haches plates des collections du musée Dobrée à Nantes et du musée d'archéologie nationale (MAN) à Saint-Germainen-Laye a permis de retrouver une hache portant l'indication « Saint-Père-en-Retz, Loire-Inférieure ». Le mobilier qui aurait été trouvé dans un " dolmen » de cette commune (deux artefacts en or et deux haches plates en cuivre / alliage cuivreux) est souvent considéré comme emblématique de la culture campaniforme et abondamment cité dans la littérature. Les artefacts ayant été collectés par F. Parenteau, premier conservateur du musée départemental d'archéologie de Loire-Atlantique, la consultation du très riche fonds de cet érudit a permis d'y retrouver certains documents inédits qui apportent de nouveaux éléments concernant cet ensemble. Il en ressort que les objets n’ont pas été découverts par Parenteau lui-même comme il l'écrivit a posteriori en 1872 (musée des beaux-arts de Nantes, 1872, p. 36), mais achetés en seconde main à un orfevre de Nantes. Les notes de Parenteau permettent de se rendre compte que la localisation (Saint-Père-en-Retz) et le contexte (" dolmen ») du dépôt sont peu sûrs et ce, dès la découverte même. Par la suite, les diverses publications se sont parfois livrées à des ajouts fantaisistes de matériel. Des dessins inédits issus du fonds Parenteau complètent la documentation de cet ensemble, mais mettent aussi fortement en doute la provenance de la hache plate du MAN, tout en permettant d'y rattacher une des haches du musée Dobrée. Au final, les nouveaux éléments rassemblés indiquent que l'ensemble connu et mentionné à profusion est extrêmement douteux à la fois aux points de vue localisation et contexte, et que la consultation des vieilles archives du XIX ${ }^{\mathrm{e}}$ siècle s'avère souvent d'une aide inestimable pour l'étude des collections anciennes.
\end{abstract}

\begin{abstract}
Whilst studying flat axes in the collections of the Dobrée Museum in Nantes and the National Archaeology Museum in Saint-Germain-en-Laye, it was found that one of the axes had the mention "Saint-Père-en-Retz, Loire-Inférieure" written on it. Found in the so-called "dolmen of Saint-Père-enRetz" along with another axe and two gold artefacts, this material is often considered as typical of the Beaker culture and has thus been covered in a large number of papers and books. The objects were collected by F. Parenteau, the first curator of the local archaeology museum, the study of its private archives kept in the Dobrée Museum provided unpublished documents concerning this find. The objects were not discovered by Parenteau himself as he wrote in a publication that came out much later in 1872 (Musée des Beaux-arts de Nantes, 1872, p. 36), but were bought second-hand from a goldsmith in Nantes. Parenteau's notes lead us to understand that both the location (Saint-Père-en-Retz) and the context ('dolmen') are not verified. The subsequent publications sometimes contained fanciful artefacts. Some previously unpublished drawings from the Parenteau archives do complete the documentation we have while at the same time cast a doubt over the provenance of the Saint-Germain-en-Laye axe. It also provides us with an opportunity to put forth a new hypothesis as to the provenance of one of the axes of the Dobrée Museum. To conclude, the new elements gathered here show that both provenance and context are extremely doubtful, and that therefore the study of the 19th century archives can often be of invaluable assistance when one works on old collections.
\end{abstract}

Mots clés : hache plate, Parenteau, dolmen, mobilier en or, gargantilla, Chalcolithique, Bronze ancien, Saint-Père-en-Retz, musée Dobrée.

Keywords: flat axe, Parenteau, dolmen, gold material, gargantilla, Chalcolithic, Early Bronze Age, Saint-Père-en-Retz, Dobrée Museum.

\footnotetext{
${ }^{a}$ Doctorant, Université de Paris I, UMR8215 - Trajectoires.

${ }^{b}$ Conservateur du patrimoine, directrice du musée Dobrée et des sites patrimoniaux départementaux, Grand-Patrimoine de Loire-Atlantique, conseil général de Loire-Atlantique.
} 


\section{INTRODUCTION}

Lors d'une étude sur les haches plates des collections du musée Dobrée à Nantes et du musée d'archéologie nationale à Saint-Germain-en-Laye les deux haches mentionnées dans la première publication sur le mobilier du "dolmen " de Saint-Père-en-Retz (Loire-Atlantique; musée des beauxarts de Nantes, 1872, p. 36) ont pu être retrouvées dans les réserves, même si l'attribution de celle de Saint-Germain-enLaye pose problème, et ce, malgré la mention explicite de la provenance sur l'objet lui-même (cf. infra), et que celle du musée Dobrée n'est pas plus certaine car elle ne repose que sur des documents anciens. L'étude de ces haches est également l'occasion de se pencher en détail sur cet ensemble censé provenir d'un " dolmen ». En effet, outre les haches, deux éléments en or y auraient été découverts (Parenteau, 1878, p. 25-26) et, si ces derniers ont rapidement été dispersés dans des collections privées, ils sont abondamment cités et reproduits dans la littérature avec parfois quelques ajouts fantaisistes de mobilier. La consultation du fonds Parenteau au service de documentation du musée Dobrée à Nantes permet d'apporter un nouvel éclairage sur cet ensemble souvent considéré comme emblématique du campaniforme, mais qui demeure assez douteux, ainsi que le lieu même de découverte.

\section{Fortuné Parenteau, vie et collections}

Fortuné Parenteau (Luçon, 7 avril 1814 - Pouzauges, 10 septembre 1882) compte parmi les pionniers de l'archéologie dans le Grand Ouest. Élevé dans une famille bourgeoise, dont l'origine est à la fois vendéenne et poitevine, il entreprend à partir de 1830 dix années d'études au Collège de France. De retour à Luçon, et fort de cet important bagage en sciences humaines, il commence alors à collectionner. D'abord intéressé par l'orfèvrerie et la numismatique, il se passionne ensuite pour ce que l'on appelle aujourd'hui l'archéologie nationale, dans le sillage des nombreuses sociétés savantes constituées au cours du XIX $^{\mathrm{e}}$ siècle. En 1851, il se marie et s'installe à Nantes. Il devient membre de la société historique et archéologique de Nantes et la Loire-inférieure, avant d'être admis au bureau de l'association en 1854. Le rythme soutenu des découvertes archéologiques, lié aux travaux d'urbanisme de la ville et de ses environs, lui permet d'enrichir sa collection et il publie d'ailleurs régulièrement ses trouvailles, faisant preuve d'un véritable talent de dessinateur. Il fouille à Pouzauges, Rezé et suit les chantiers urbains de Nantes qui remontent de nombreux vestiges archéologiques. Il croque inlassablement le mobilier qu'il peut observer, et nous lui devons la représen- tation de quelques œuvres emblématiques de l'actuel musée Dobrée. À titre plus personnel, il se documente sans cesse, dessine et compulse des données sur les thématiques archéologiques qui retiennent son attention, tout en guettant les trouvailles pour enrichir sa propre collection (Santrot, 2001). C'est dans ce contexte qu'il acquiert le mobilier de Saint-Père-en-Retz, découvert en 1851 et qui avait transité par un orfèvre de Nantes avant d'être vendu sur le marché parisien. On apprend d'ailleurs, par Parenteau lui-même, qu'il achète par la suite la bague en or auprès d'un orfèvre nantais, pour la réunir aux autres pièces a priori issues de la fouille du «dolmen».

En 1856, le volume des collections rassemblé par la société archéologique est tel qu'il faut quitter le local trop exigu de la rue du Moulin pour la chapelle de l'Oratoire, où un transfert des objets est organisé. Concomitamment, Parenteau est chargé de la rédaction du premier catalogue des collections de la société, appelées à former le noyau des collections archéologiques du futur musée Dobrée (Parenteau, 1856). La société historique et archéologique de Nantes et de la Loire-inférieure donne en effet ses collections au département en 1861. Ce fonds sera réuni en 1895 au legs Dobrée pour former le musée actuel (musée Dobrée, fonds Parenteau). Parenteau devient le premier conservateur du musée départemental en 1861. Il poursuit les acquisitions pour le compte de l'institution, tout en continuant la recherche sur ses propres collections. En 1869, il publie un second catalogue, le Catalogue du musée départemental d'archéologie. Les dernières années de sa vie sont consacrées à la rédaction du catalogue complet de sa collection archéologique, qu'il publie en 1878 . Peu avant sa mort en 1882 , Parenteau fait don de sa collection au musée Dobrée, qui conserve aujourd'hui également d'importantes archives, notamment des carnets de sa main, riches de très nombreux dessins d'objets archéologiques.

\section{ConteXte, MOBILIER ET LIEU DE DÉCOUVERTE, UNE MISE AU POINT}

Si le "mobilier en or du dolmen de Saint-Père-enRetz " (Loire-Atlantique) est très bien documenté par une bibliographie abondante (musée des beaux-arts de Nantes, 1872, p. 36; Parenteau, 1878, p. 25; Chantre, 1875-1876, p. 106-107; Déchelette, 1910, no 460, p. 72 ; Briard et L'Helgouach, 1957, p. 10 et p. 15; Galan et Soutou, 1959, p. 603; Riquet et al., 1963, p. 88; Briard, 1965, p. 68; Eluère, 1977 , p. $401-402$; 1982, p. 135, p. 266-267; Joussaume et Pautreau, 1990, p. 350), la connaissance du contexte exact de découverte ainsi que celle du reste du mobilier demeure au mieux imprécise, au pire erronée. Il 
faut d'ailleurs noter que l'iconographie, répétée à l'envi, du mobilier en or du « dolmen » de Saint-Père-en-Retz provient exclusivement de la planche de Fortuné Parenteau de 1878 (fig. 1,10), le mobilier ayant été dispersé et perdu depuis.

L'étude des deux haches mentionnées en introduction est l'occasion de faire le point sur le mobilier, les conditions et le lieu de découverte; la consultation du fonds Parenteau au service de documentation du musée Dobrée à Nantes permettant d'apporter de nouvelles informations sur ces différents points.

Une reprise exhaustive des différents inventaires publiés semble nécessaire afin d'essayer de comprendre leur évolution, et subséquemment de constater qu'au mieux, une certaine confusion règne sur cet ensemble. L'utilisation du fonds Parenteau permet de se faire une idée plus précise, sans pour autant apporter de nouveaux éléments vraiment probants quant à l'ensemble. Le réexamen approfondi des inventaires se fera par ordre chronologique; par la suite, les éléments inédits du fonds Parenteau seront exposés.

À notre connaissance, la première mention publiée de ce mobilier se trouve dans le catalogue raisonné de l'exposition au musée des beaux-arts de Nantes en 1872, dans une notice rédigée par Fortuné Parenteau : "L'âge de la pierre polie arrivé à son apogée, l'or, le premier de tous les métaux, ne tarde pas à paraître; nous le rencontrons sous les dolmens de Quimper, puis sous les dolmens de Plouharnel, et, enfin, joint aux haches en cuivre calquées sur les haches en pierres polies de la dernière époque; sous les roches druidiques de Saint-Père-en-Retz. Cette dernière découverte, faite par moi en 1851, comprenait un bandeau ou diadème, comme celui de Plouharnel, un léger ruban d'or, ou tonia, pour la coiffure, et deux haches rugueuses en cuivre. " (Musée des beaux-arts de Nantes, 1872, p. 36). On peut noter d'emblée que Parenteau affirme ici que c'est bien lui-même qui a fait la découverte, le mobilier se composant de deux haches et de deux éléments en or.

Peu de temps après c'est $\mathrm{E}$. Chantre qui cite cet ensemble : "En 1831, sous des roches druidiques. Deux haches avec un bandeau [coll. Charvet] et un léger ruban d'or. " (Chantre, 1875-1876, p. 106-107.) Trois ans après la première publi- cation, la date de découverte diffère déjà, même si c'est vraisemblablement une erreur d'édition; le problème est qu'elle a été reprise par la suite par Déchelette (cf. infra)...

La publication principale (et de référence) avec les dessins des objets est réalisée par Parenteau en 1878 dans son inventaire archéologique :

"Au mois de juin de l'année 1851, on découvrit sous un dolmen de la commune de Saint-Père-en-Retz (Loire-Inférieure), des bijoux d'or et deux haches rugueuses en cuivre, haches calquées sur les haches en pierres polies de la dernière époque, même grandeur, même épaisseur. [...]

"- diadème ou bandeau en or martelé allié à de l'argent dans la proportion d'un $8^{\text {ime }}$; 18 stries, et deux bouts arrondis. Poids, 190 grammes; long., $0^{m} 46$; hauteur, $0^{m} 06$.

"- Tonia en or, ruban terminé par deux petits disques. Poids, 10 grammes. (Fragmenté.)

"- deux haches rugueuses en cuivre. " (Parenteau, 1878, p. 25-26).

Il faut noter qu'il s'agit là de la première apparition du mot "dolmen ", sans qu'il soit possible de savoir s'il s'agit d'une simple interpolation de Parenteau à partir des « roches druidiques » initialement mentionnées.

Une trentaine d'années plus tard, c'est au tour de Déchelette, dans son ouvrage fondamental sur l'âge du Bronze, de citer à nouveau l'ensemble de Saint-Père-enRetz : " 460. Saint-Père-en-Retz (sous des roches druidiques). - $1831-2$ haches, 1 bandeau [coll. Charvet] et un léger ruban d'or (C.).» (Déchelette, 1910, Appendice I, p. 72). Il semble, presque mot pour mot, se référer ici à Chantre plus qu’à Parenteau (les deux étant cités dans sa bibliographie), ce dernier livrant une description bien plus précise.

Il faut par la suite attendre la fin des années cinquante pour que le mobilier soit à nouveau mentionné par J. Briard et J. L'Helgouach : " Les plus beaux bijoux découverts sont sans aucun doute les célèbres bracelets du dolmen de Rondossec en Plouharnel (Morbihan). Trouvés dans un vase, ils se caractérisent par le découpage de leur partie interne en fines lanières. D'autre part, l'un d'eux est ornementé sur les côtés. Le système d'agrafage, très simple, est réalisé par le replie-

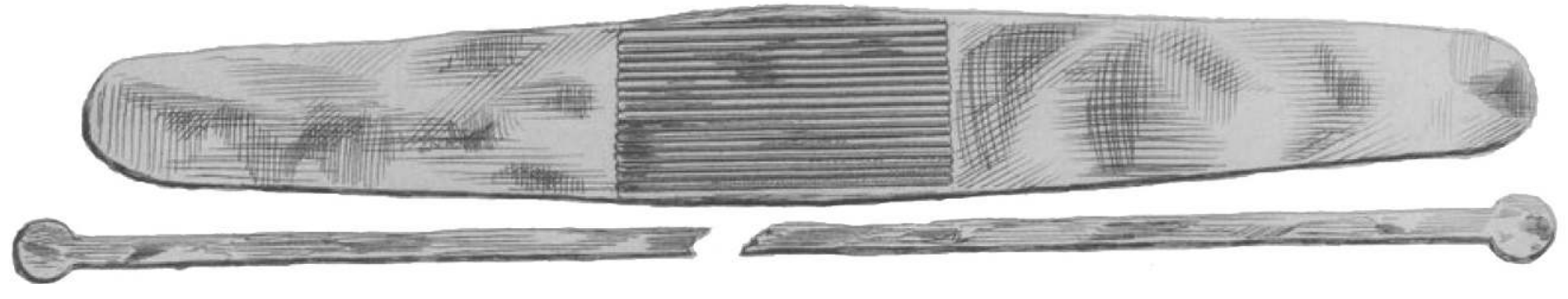

Figure 1 : Mobilier en or du dolmen de Saint-Père-en-Retz (Loire-Atlantique), sans échelle. D'après Parenteau, $1878, \mathrm{Pl} .61, \mathrm{n}^{\text {os }} 1$ et 2. Figure 1: Gold artefacts from the dolmen of Saint-Père-en-Retz (Loire-Atlantique) no scale. 
ment des extrémités. Presque semblable, mais légèrement plus grand et simplement strié est un bracelet trouvé dans un dolmen de Saint-Père-en-Retz (Loire-Inférieure), associé à des haches plates en cuivre. Dans le même monument était une petite bague de technique tout à fait semblable à celle du bracelet. Elle s'agrafe par un crochet pénétrant dans l'anneau de l'autre extrémité. Citons tout de suite, dans cette même sépulture, deux fragments d'un torque à palettes. " (Briard et L'Helgouach, 1957, p. 10.)

Le "diadème ou bandeau » devient maintenant un simple bracelet, le ruban d'or se transforme en torque à palette (de $10 \mathrm{~g}$ si l'on continue à se fier à Parenteau...), et en outre une bague fait maintenant son apparition dans l'inventaire. Cette bague par contre est conservée au musée Dobrée à Nantes (inv. $\mathrm{n}^{\circ}$ 882.1.437), cet objet ayant été publié par Parenteau (1878, Pl. 11, nos 1 et 2; fig. 2).

La question de son origine demeure peu évidente, Parenteau écrit à son sujet (ibid., p. 27) : "Large bague d'or à crochet, couverte de filets en relief, comme le bandeau de Saint-Père-en-Retz. Elle arriva à Nantes, chez un orfèvre de la rue d'Orléans, peu de jours après la découverte dont je viens de rendre compte. Même provenance. Poids, 4 grammes."

Que faut-il comprendre de la mention " même provenance »? Vient-elle du prétendu " dolmen » de Saint-Pèreen-Retz tout comme le bandeau, le ruban et les haches plates, ou bien s'agit-il simplement de la même commune? Le fait que Parenteau mentionne très précisément ce qui a été trouvé dans le "dolmen " $(1878$, p. 25$)$ sans évoquer cette bague, et que, de plus, elle soit illustrée sur une planche à part plaident plutôt dans le sens d'une découverte indépendante. Néanmoins, on ne peut exclure le fait que ce petit objet ait été trouvé dans le "dolmen ", à la suite des premières recherches, sa petite taille ayant pu, dans un premier temps, le soustraire à l'attention des fouilleurs. Dans tous les cas il faut également noter ici la rigoureuse exactitude du dessin de Parenteau, il figure même douze stries sur les treize présentes, ce qui ne peut qu'inciter à faire confiance à ses autres dessins.
Deux ans après cette publication une nouvelle mention de ce "dolmen " est faite, mais en insistant cette fois-ci sur la bague en or : "Une étroite parenté typologique semble exister entre la boucle d'oreille de Mios (diamètre : 2,7 cm, largeur : 0,5 cm) et une "bague" en or trouvée dans le dolmen de Saint-Père-en-Retz, Loire-Inférieure (J. Briard et J. L'Helgouach, Chalcolithique, Néolithique secondaire, survivances néolithiques du Bronze ancien en Armorique, Rennes, 1957, p. 10-12 et pl. 6).

"Cette "bague" (diamètre : 2,5 cm, largeur : 0,9 cm) est constituée par un simple ruban métallique fermé par un crochet qui s'introduit dans un trou placé à l'extrémité opposée. »Comme le pensent les auteurs (ibidem, p. 15), il ne s'agit pas d'un mobilier funéraire contemporain de l'érection du dolmen mais d'une " cachette " postérieure, attribuée à l'âge du Bronze ancien. Malgré la présence de deux haches à rebord qui ne sont pas nécessairement associées à la "bague ", nous y verrions plutôt un dépôt votif ou rituel de l'âge du Fer, contemporain du tumulus de Mios. Un «bracelet strié " de même type, accompagnait la "bague ": il serait souhaitable que ce bijou, en or également, soit publié (Galan et Soutou, 1959, p. 603).

Ici, la bague est attribué à l'âge du Fer et serait donc un dépôt postérieur à celui du bracelet/bandeau et des deux haches (qui deviennent maintenant des haches à rebord...), le ruban, quant à lui, disparait de l'inventaire du mobilier.

En 1963, l'inventaire du mobilier s'étoffe de perles d'or, d'un vase campaniforme et de flèches "assez bretonnes ", semblant donc confirmer un mélange assez prononcé de différents mobiliers, mais sans qu'il soit possible de retrouver l’origine de ces éléments intrusifs.

"Enfin, un dolmen de Saint-Père-en-Retz a livré des perles d'or, une bague ou boucle d'oreille du même métal rappelant les boucles du tumulus de Hills Field (Radney, Berks) associées à un campaniforme de classe BI (affinités continentales) et à des flèches assez bretonnes. Le même monument de Saint-Père-en-Retz contenait aussi un torque à palettes en or. Comme il y avait aussi deux haches plates, le tout se classe probablement au Chalcolithique ou début du Bronze
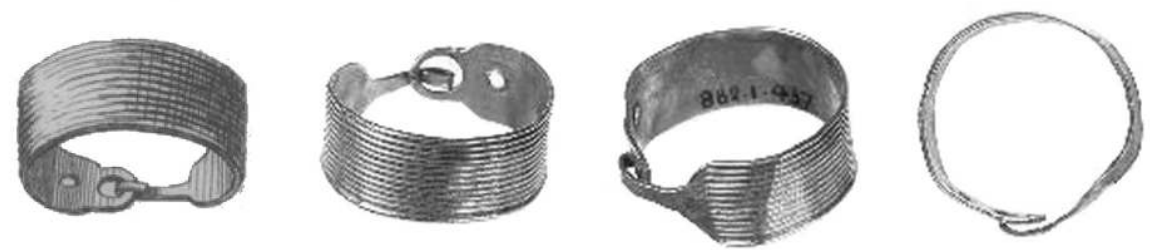

0

$5 \mathrm{~cm}$
Figure 2 : Bague en or provenant de Saint-Père-en-Retz. D'après Parenteau, 1878, Pl. 11, nº 1 et cliché Hervé Neveu-Dérotrie, musée Dobrée et sites patrimoniaux - Grand patrimoine de Loire-Atlantique.

Figure 2: Gold ring from Saint-Père-enRetz. 
ancien (F. Parenteau, Inventaire archéologique, Nantes, 1879 [sic]).» (Riquet et al., 1963, p. 88).

Jacques Briard décrit à nouveau le mobilier de Saint-Pèreen-Retz dans son ouvrage de référence en 1965 : «Enfin le dépôt de Saint-Père-en-Retz (L.-A.) associait deux haches en cuivre à une "gargantilla" ou collier en or de type portugais. Malheureusement, F. Parenteau (1878) ne donne qu'un dessin sommaire des haches qui empêche de les classer dans une catégorie précise. " (Briard, 1965, p. 68.)

Les éléments campaniformes disparaissent à présent, mais le ruban en or, lui aussi, semble ne plus faire partie de l'ensemble.

C'est enfin Christiane Eluère qui remet à plat l'ensemble en 1982 dans son étude sur les ors préhistoriques : «Le dépôt de Saint-Père-en-Retz est d'un grand intérêt parce que, d'une part, comme à Kérivoa, il comporte plusieurs types d'ors rassemblés (ici, collier à lamelles découpées et torques à palettes) et, d'autre part, ces ors sont associés à des haches plates qui confirment la place chronologique que par ailleurs on leur attribue. " (Eluère, 1982, p. 135.)

L'inventaire du même ouvrage (p. 266-267) précise un lieu-dit de découverte (Port Saint-Père) et indique :

"- [Type de découverte, année] : Dolmen, en 1851;

[Lieu de conservation actuel du mobilier et ancienne collection] : Disparu?;

«- [Bibliographie principale] : Parentean 1878, p. 25-26, pl. 61, no 1-2; Briard, 1965, p. 71-74; Eluère 1977, p. 401;

«- [Description du mobilier] : Deux fragments d'un torque à palette : L. $45 \mathrm{~cm}$ environ; $10 \mathrm{~g}$./Collier à décor de lamelles découpées : L. $46 \mathrm{~cm}$ environ; $190 \mathrm{~g}$ (le collier présente $1 / 8$, soit $12,5 \%$ d'Ag);

«- [Description sommaire du contexte signalé] : deux haches plates en cuivre et, selon Riquet, Guilaine, Coffyn (1963), p. 88 : pointes de flèche en silex "assez bretonnes" et un vase campaniforme;

"- [Datation proposée] : B.A.;

" Fig. $79 n^{\circ} 2$ et 145."

Si le mobilier campaniforme réapparaît mais avec quelques précautions, on revient néanmoins vers l'inventaire initial de Parenteau.

Parmi tous les éléments publiés, on peut retenir, dans un premier temps seulement, la localisation et le type de contexte : un "dolmen " ou des " roches druidiques " à Saint-Père-en-Retz (ces variations terminologiques sont cependant à relativiser vu le contexte de l'époque), et pour le mobilier : deux haches plates en cuivre et deux éléments en or (ruban et collier/bracelet). Pour essayer de clarifier la situation, le fonds Parenteau au centre de documentation du musée Dobrée à Nantes a été consulté afin de voir si des relations de fouilles (Parenteau écrit en 1872 que c'est lui-même qui a fait la découverte) ou bien des notes concernant cette découverte pouvaient être retrouvées, et de fait, plusieurs mentions et dessins inédits l'ont été. Le fonds conserve, entre autres, ses albums personnels, véritables carnets de terrain dans lesquels il note ses découvertes, ses impressions et où il réalise de nombreux croquis souvent admirablement bien faits.

La première mention (inédite) de l'ensemble dit de SaintPère-en-Retz se retrouve dans l'un de ces albums, daté de $1858 / 1862$, on y voit notamment deux dessins originaux du mobilier en or (fig. 3) ainsi qu'une brève description (il s'agit donc de notes de travail que nous reproduisons in extenso) :

"Découverte de Pornic, Juin 1851:

"Longueur du grand torque: $0^{\mathrm{m}}, 40^{\mathrm{cm}}$

"Largeur du grand torque: 0 m, 13 cm

"Poids = 195 grammes, petit torque compris

"Diamètre $=0^{\mathrm{m}}, 13^{\mathrm{cm}}$

"Circonférence $=0^{\mathrm{m}}, 40^{\mathrm{cm}}$

" Hauteur $=0^{\mathrm{m}}, 06^{\mathrm{cm}}$

"Petit bandeau : longueur: $0^{\mathrm{m}}, 30$; largeur: $0^{\mathrm{m}}$ cinq millimètres aux petites plaques circulaires aux extrémités.

«NB: nombre des filets 18, coupe d'un filet: le tout travaillé au marteau, sans soudure. Les deux haches en bronze grossier très rugueuses, coulées grossièrement dans du sable affectaient la forme de l'âge de Pierre, elles n'avaient point été aiguisées, neuves.

"NB : cette découverte, identique à celle de Plouharnel, Morbihan, fait [sic] à Nantes chez Me Delambre Rue Contrescarpe; l'or était allié à l'argent, je crois ces objets antérieurs à L'âge du Fer. À l'époque de transition. Sur le refus de Mr Lenormant de la Bibliothèque, ils furent vendus au poids. " (Parenteau [a], p. 73).
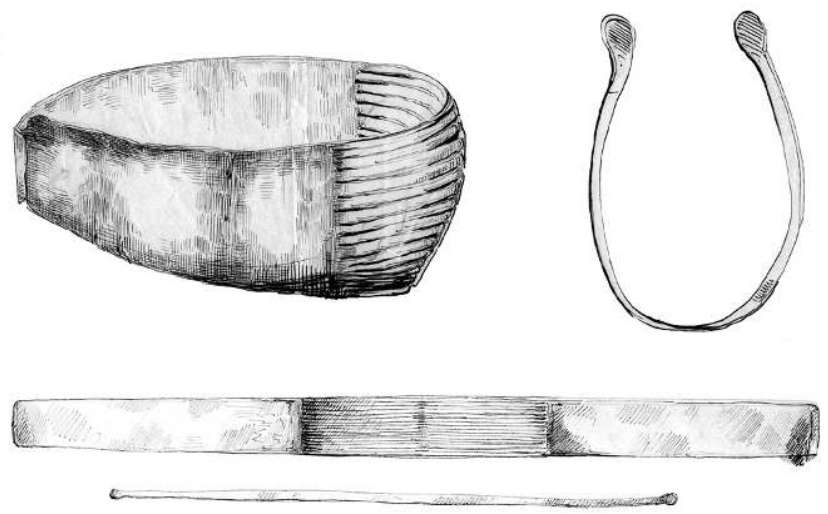

Figure 3 : Deux dessins inédits par F. Parenteau du mobilier en or (Parenteau [a], p. 72 en haut; p. 73 en bas. Cliché Chantal Hémon, musée Dobrée et sites patrimoniaux - Grand patrimoine de Loire-Atlantique.

Figure 3: Two unpublished drawings by F. Parenteau of the two gold artefacts. (Parenteau [a], p. 72 above; $p .73$ below). 
De cette première description, on se rend compte d'emblée que contrairement à ce que Parenteau écrivait en 1872, ce n'est pas lui qui a fait la découverte, mais qu'il a vu les objets chez une certaine $\mathrm{M}^{\text {me }}$ Delambre à Nantes, qui n'est en plus certainement pas la personne qui a trouvé les objets. Le lieu de découverte, s'il est ici exact, n'est pas Saint-Pèreen-Retz, mais Pornic (commune certes limitrophe), cette imprécision est compréhensible lorsqu'on sait que Parenteau n'a pu voir les bijoux qu'en troisième main après l'inventeur initial et la marchande horlogère, $\mathrm{M}^{\mathrm{me}}$ Delambre. Nulle mention ici de quelconques roches druidiques (musée des beaux-arts de Nantes, 1872, p. 36) ou de "dolmen " (Parenteau, 1878, p. 25), le contexte n'étant tout simplement pas mentionné. Enfin, il n'est nullement fait mention de la petite bague en or dans ce premier inventaire (rédigé au plus tôt en 1858, soit sept ans après la découverte). On peut également noter que les noms des rues concernant le bijoutier Nantais diffèrent (rue d'Orléans et rue Contrescarpe), indiquant peut-être qu'il s'agit de deux bijoutiers différents, ce qui ne fait que renforcer l'hypothèse d'une arrivée intempestive de la bague dans le lot. Au final, seul le mobilier initial est confirmé, de même que les dimensions et les poids à quelques nuances près.

Ce n'est que vingt-sept ans après la découverte que Parenteau publie son inventaire archéologique (1878). De nombreuses notes préparatoires à ce travail sont disponibles au musée Dobrée dans le fonds Parenteau, et parmi elles, certaines concernent le mobilier dont il est question ici.

"Découverte de Saint-Père-en-Raiz - Loire-Inférieure - Mai 1851

"Sous un dolmen de Saint-Père-en-Raiz, Loire-Inférieure, on découvrit au mois de mai ou de juin 1851, un diadème ou bandeau d'or d'une longueur de $0^{n} 46^{c}$ sur une hauteur de $0^{m}$, $06^{\mathrm{cm}}$ du poids de 190 grammes, un toenia, sorte de bandeau terminé par deux petits disques et deux haches celtiques en cuivre très primitives. Ces curieux objets restèrent plus d'un mois chez un orfevre de la rue Contrescarpe à Nantes, puis, furent emportés et vendus à Paris. L'or était allié à l'argent, produit sans doute du lavage des sables auriferes des rivières de l'Armorique. Ce bandeau, ayant au centre un certain nombre de filets détachés et arrondis ressemblait à ceux de Rennes, de Niort et de Plouharnel. Plus tard la bague en or de ma collection, bague à crochets avec filets arriva chez les orfevres de Nantes et provient, je n'en saurais douter de la même découverte. Depuis cette époque jusqu'à nos jours les haches en cuivre trouvées isolément furent recueillies. "(Parenteau [b], p. 249).

La provenance de la bague est donc, et ce malgré les dires de l'auteur, on ne peut plus douteuse, il s'agit là de son avis qui n'est motivé semble-t-il que par la matière similaire des objets! La commune change désormais de Pornic à Saint-Père-en-Raiz, et le " dolmen » apparaît. Cependant, quelques pages plus tard, la commune de Saint-Père-en-Retz n'est même plus certaine :

"Bandeau gaulois en or circonférence quarante-six centimètres, largeur six centimètres. Poids cent quatre-vingt-dix grammes. Or allié à l'argent. Petit torque poids six grammes.

"Trouvé dans les environs de Paimbouf ou de Saint-Père-enRaiz. Juin 1851. Rive gauche de la Loire.

"J'ai vu un bandeau semblable dans le musée archéologique de la ville de Niort et un a été trouvé dans les environs de Rennes, conservé au musée de cette ville il existe un fac-simile au musée de la société archéologique de Nantes.

"NB: il y avait avec le bandeau ci-dessus deux haches en bronze ayant la forme des haches en silex de nos collections. Ces plaques different des hausse-cols publiés par M. Decaumont et sont, je crois, particulières à nos provinces de l'Ouest. Bretagne et Poitou. La petite bague de ma collection paraît être d'une fabrication analogue et de même provenance elle a été apportée en Juin 1851 chez un orfevre de la rue d'Orléans.

"Métallurgie d'un peuple [?], bijouterie tout à fait primitive il semble que l'ouvrier ignorait l'art de souder, que le martean et un burin grossier en silex sans doute aient été les seuls outils à la disposition de l'ouvrier. Le procédé [?] de fermeture de la bague semble l'indiquer. Pour ce qui regarde le bandeau ci-dessus je n'ai jamais pu découvrir comment on pouvait sen servir et à quel usage il était destiné, comment le fixait-on sur la toile ou sur le cuir?

$"+$ le collier de Niort porte quatorze stries et un petit ornement pointillé encadrant tout le bandeau, les deux extrémités sont percées de deux trous, mais sont-ils de l'époque?" (Ibid., p. 252)

Il faut noter que Parenteau ne cite aucunement ses sources à propos du bandeau trouvé dans les environs de Rennes et dont un fac-similé aurait existé au musée de Nantes; à part cette mention, il n'a été trouvé nulle part mention de cet objet dans la littérature.

\section{LA QUESTION DES HACHES PLATES}

\section{La hache plate de Saint-Germain-en-Laye}

La hache plate retrouvée dans les réserves du musée d'archéologie nationale et portant l'inscription «Saint-Père-enRetz (L. I.) " ne semble pas avoir été étudiée et publiée depuis le dessin qu'en avaient fait les frères de Mortillet en 1903 (Pl. XCVIII, no 1314; fig. 4). Il faut d'ailleurs noter la relative exactitude du dessin des de Mortillet (fig. 4 et 5); au $\mathrm{XIX}^{\mathrm{e}}$ siècle, beaucoup de leurs contemporains publiaient des croquis n'évoquant que vaguement l'objet initial (Gandois et Barthet, 2013, fig. 2 à 7)... 


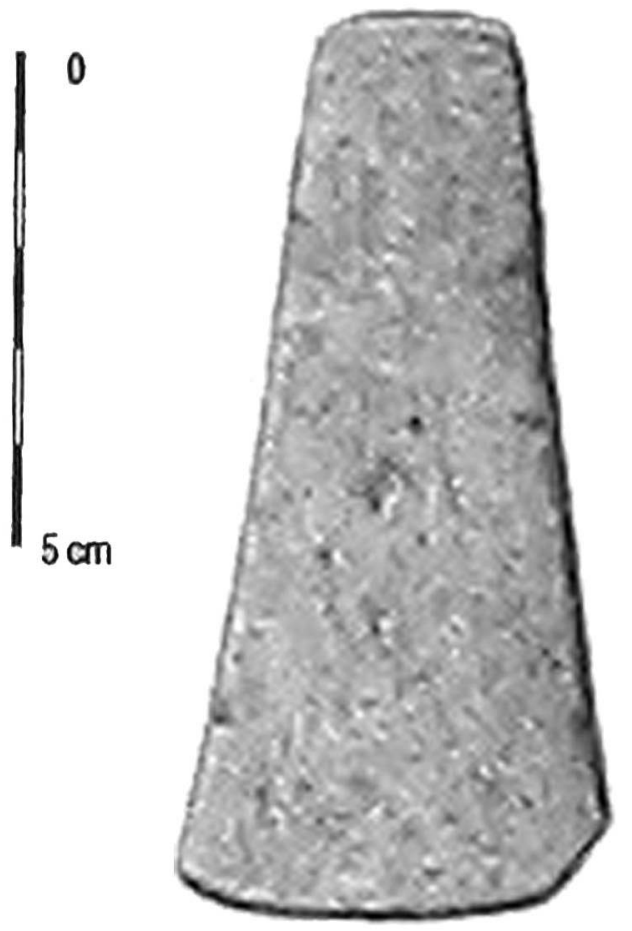

Figure 4 : Hache plate du MAN, indiquée comme provenant du dolmen de Saint-Père-en-Retz. D’après de Mortillet, 1903, $\mathrm{n}^{\mathrm{o}} 1314$, pl. XCVIII).

Figure 4: Flat axe said to originate from the dolmen of Saint-Pèreen-Retz.

La trace même de cette hache semblait avoir disparu au $\mathrm{xx}^{\mathrm{e}}$ siècle, J. Briard et J. L'Helgouach ne citant pas de lieu de conservation dans leur inventaire des haches plates en Armorique (1957, p. 65). Or, cet objet est bien entré dans les collections du MAN en septembre 1875 (Inv. no 22635), suite au don qu'en a fait M.-F. Parenteau, conservateur du musée archéologique de Nantes, par l'intermédiaire de M. G. de Mortillet. Il est mentionné dans l'inventaire comme " hache en cuivre, coin sans rebords, de Saint-Pèreen-Retz (Loire-Inférieure) ", G. de Mortillet l'avait pour sa part décrit ainsi : "Hache plate en cuivre, sans tranchant, probablement lingot. Saint-Père-en-Retz (Loire-Inférieure) » (De Mortillet, 1903, légende $\mathrm{n}^{\circ}$ 1314, pl. XCVIII). Description de l'objet (fig. 5).

Il s'agit donc d'une hache plate en cuivre/alliage cuivreux (demande d'analyse élémentaire en cours) de forme générale trapézoïdale et de profil fusiforme. Elle est recouverte d'une patine vert-brun assez homogène, quelques légères dépressions sont visibles au centre d'une des faces. Les flancs sont très légèrement concaves, le talon rectiligne et épais, le tranchant, peu évasé et nettement asymétrique, offre un profil très épais également. La section est sub-rectangulaire, mais il faut noter qu'outre les flancs, les faces sont également légère- ment bombées. Aucune trace de mise en forme ne demeure visible, même si un impact sur un coin du talon se devine, sans qu'il soit possible de dire s'il s'agit de mise en forme ou d'utilisation (quoique la première hypothèse soit la plus probable étant donné l'épaisseur du tranchant). Néanmoins, en regardant attentivement les flancs, un léger renflement longitudinal médian pourrait être interprété comme le reste d'une bavure de coulée (fig. 6). Les traces étant extrêmement ténues, il n'est pas possible d'être catégorique, mais cette hypothèse semble cependant réaliste; si c'était le cas, ceci viendrait démontrer que cette hache a été coulée dans un moule bivalve, très vraisemblablement par le talon, comme cela se pratiquera couramment aux époques ultérieures. Malheureusement, l'absence de contexte fiable (cf. infra) ne permet pas non plus une attribution chronologique précise.

La provenance est donc ici explicitement indiquée sur l'une des faces ainsi que le numéro d'inventaire du musée avec la même écriture et la même encre, ce qui prouve que cette inscription a été faite lors de l'entrée de l'objet dans les collections, et non lors de sa découverte.

Lalbum 1858-1862 de F. Parenteau, au centre de documentation du musée Dobrée, a fort heureusement également livré deux croquis des haches présentées comme ayant la même provenance que les objets en or (fig. 7).

La forme générale des deux haches sur ce croquis, malgré l'absence d'échelle et sans mention de taille dans le texte diffère très nettement de celle de la hache du MAN, et, on l'a constaté, les dessins ou croquis de Parenteau sont, de manière générale, très fiables et ressemblants, il n'y a donc que peu de chance qu'il s'agisse du même artefact. L'hypothèse d'un mélange de collection avant que l'objet ne rentre dans les collections de Saint-Germain-en-Laye semble assez plausible, la hache ayant été donnée en 1875, soit plus de vingt ans après son acquisition par Parenteau, dont on a vu que les souvenirs pouvaient être défaillants. Mais alors d'où peut provenir l'objet du MAN si ce n'est de Saint-Pèreen-Retz/Paimbœuf/Pornic?

Une fois encore le fonds Parenteau du musée Dobrée s'avère être d'une aide inestimable, car il renferme également le croquis d'une hache plate dite en bronze (fig. 8) et présentée comme venant de Frossais, Loire-Inférieure (Parenteau [c], p. 131).

La hache représentée sur ce dessin, malheureusement sans échelle, a comme caractéristique principale une nette dissymétrie du tranchant. Or, cette dissymétrie est précisément celle que l'on retrouve sur la hache des collections du MAN et présentée comme venant de Saint-Père-en-Retz. Si l'on place côte à côte (et certes à la même échelle) le croquis de Parenteau et la hache de Saint-Germain-en-Laye, il ne fait plus de doute qu'il s'agisse bien là du même objet, certaines imperfections sont même parfaitement reconnaissables (fig. 9). 

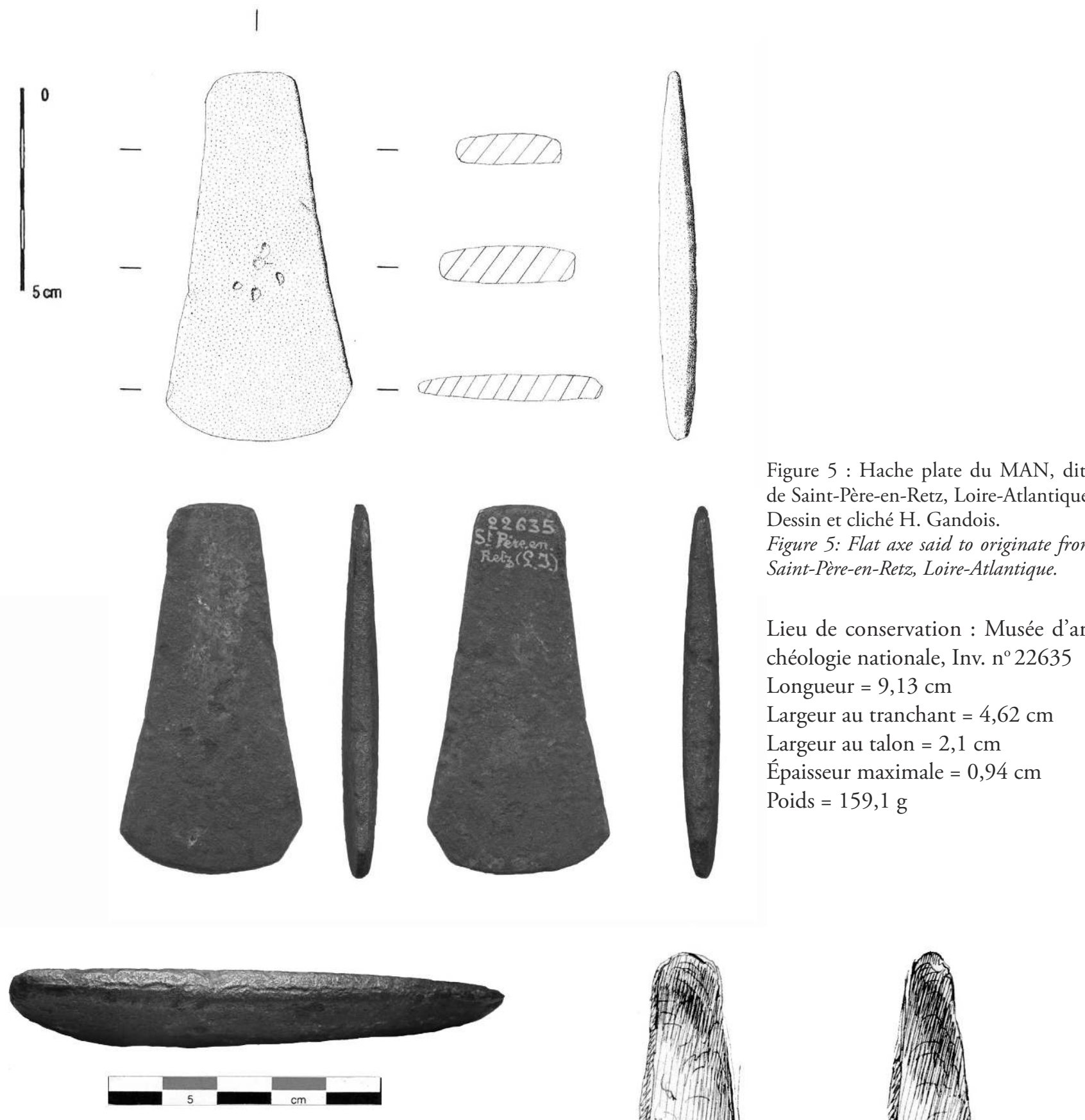

Figure 6 : Vue d'une probable bavure de coulée sur la hache plate conservée au MAN. Cliché H. Gandois.

Figure 6: view of the remains of a likely flashing on the flank of the flat axe kept in Saint-Germain-en-Laye.

Figure 7 : Croquis des deux haches associées au mobilier en or (Parenteau [a], p. 73). Cliché Chantal Hémon, musée Dobrée et sites patrimoniaux - Grand patrimoine de Loire-Atlantique. Figure 7: Sketch of the two axes found with the gold artefacts.

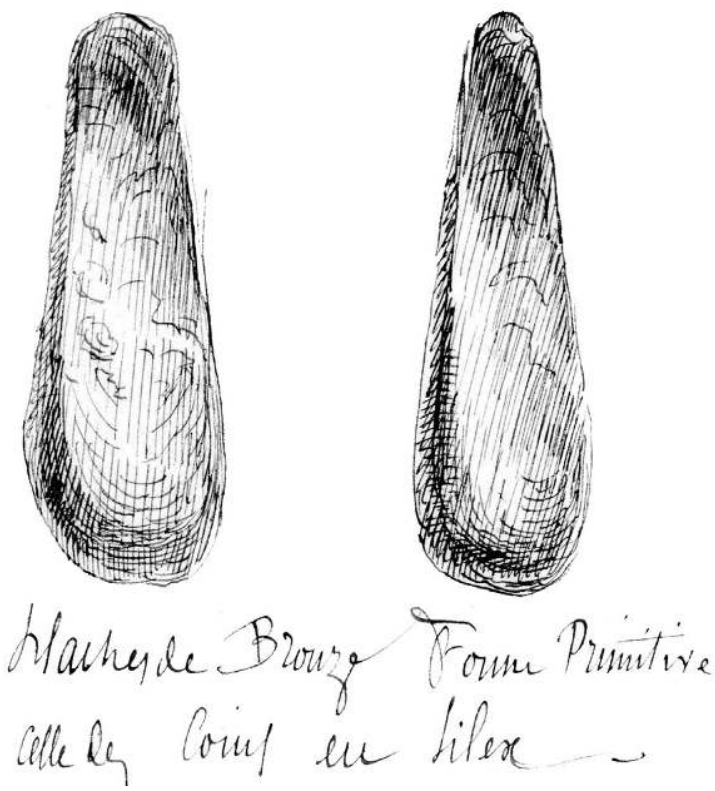




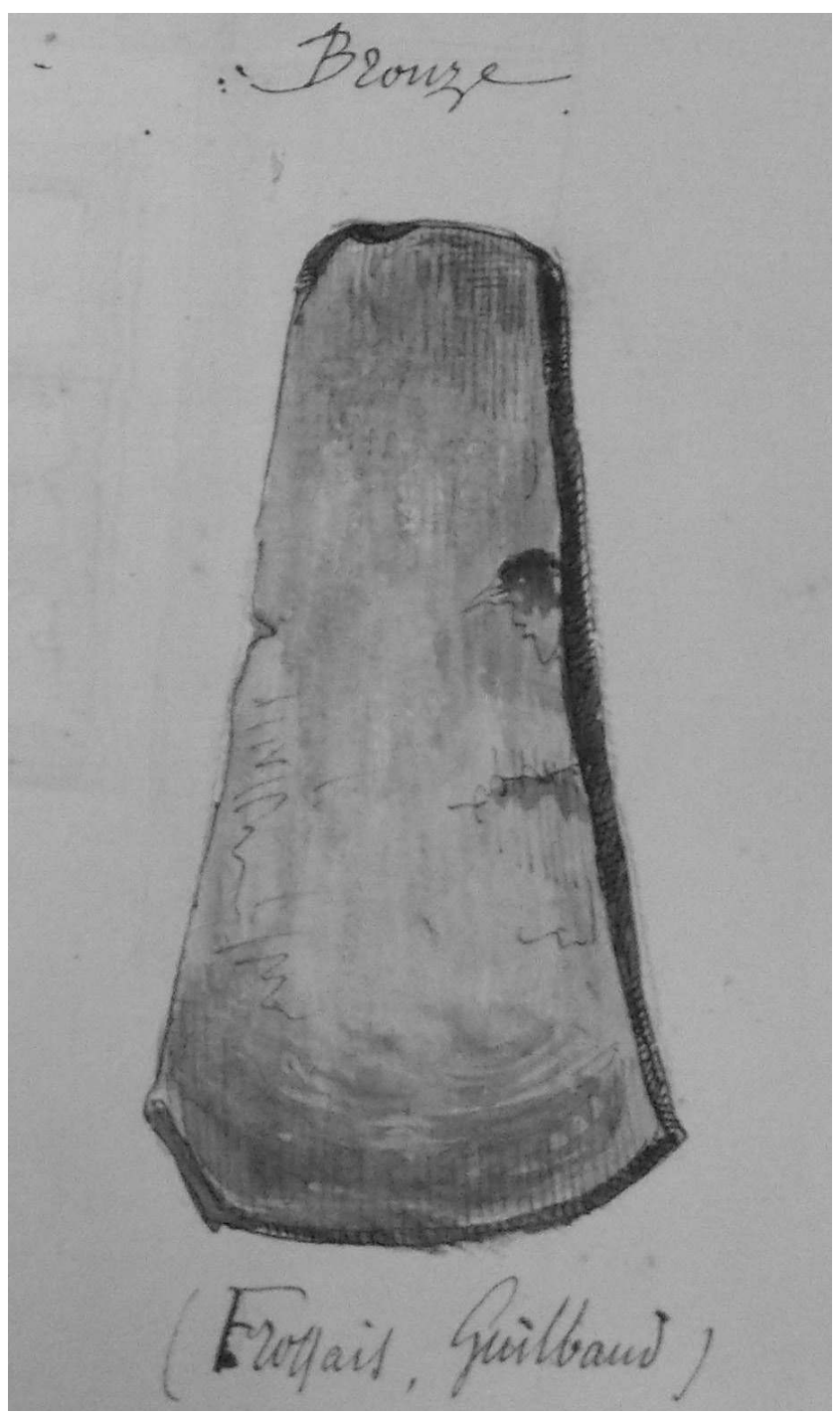

Figure 8 : Hache plate de Frossais (Loire-Atlantique; Parenteau [c], p. 131).

Figure 8: Flat axe from Frossais (Loire-Atlantique).

Il semble donc au final que la hache du musée d'archéologie nationale ne provienne pas de l'ensemble de SaintPère-en-Retz mais de la commune de Frossay (anciennement Frossais), qui d'ailleurs dépend actuellement du canton de Saint-Père-en-Retz...

\section{La hache plate du musée Dobrée}

La présence de deux haches dans l'ensemble mobilier dit de Saint-Père-en-Retz ne fait a priori pas de doute, dans la mesure où elles sont toutes deux mentionnées dès la toute première publication (musée des beaux-arts de Nantes, 1872, p. 36). Dans son ouvrage, C. Eluère (1982, fig. 145, p. 135) mentionne les haches $n^{\text {os }} 5$ et 6 de la planche 61 de

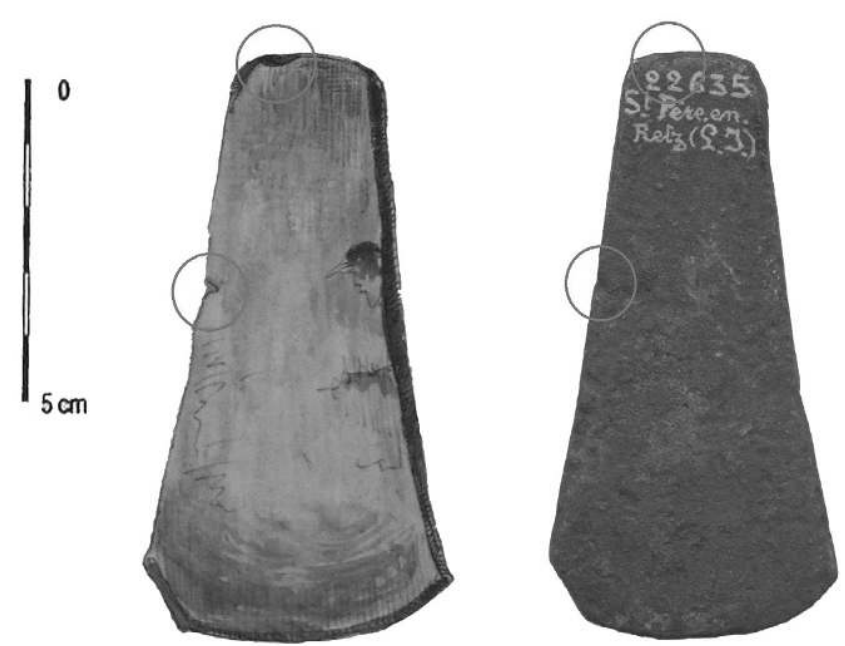

Figure 9 : Hache plate de Frossais et hache plate dite de SaintPère-en-Retz. D'après Parenteau [c], p. 131 et cliché H. Gandois. Figure 9: Flat axe from Frossais and flat said to originate from SaintPère-en-Retz.

Parenteau (1868) comme provenant du « dolmen » de SaintPère-en-Retz (fig. 10), or la $\mathrm{n}^{\circ} 5$ est spécifiquement indiquée dans la légende de la planche comme venant des environs de Nantes (Ibid., p. 26), il semble donc que l'on puisse l'exclure de l'ensemble. Le doute demeure pour la hache ${ }^{\circ} 6$; toujours dans la légende de la planche, elle est indiquée à la suite du diadème et du ruban, la provenance n'étant indiquée que pour le premier de ces deux éléments, les autres objets sur la planche ayant tous leurs origines bien spécifiées. Le fait qu'elle soit indiquée directement à la suite des deux objets en or, qui eux proviennent incontestablement du "dolmen ", de plus avec un numéro de légende ne suivant pas ceux-ci incite fortement à penser qu'elle fait partie du même ensemble. En outre, Parenteau (ibid., p. 25), dans un court paragraphe dédié à la découverte de Saint-Père-enRetz, écrit que les haches du "dolmen » étaient " calquées sur les haches en pierre polies de la dernière époque, même grandeur, même épaisseur ». Or, cette remarque est précisément reprise dans la légende de la hache $\mathrm{n}^{\circ} 6$ (fig. 10), ce qui tend à confirmer son appartenance au mobilier initial dudit « dolmen ».

La hache retrouvée au musée de Saint-Germain-en-Laye (fig. 5) ne correspond à aucune des haches figurées ici, mais lorsque Parenteau a rédigé son inventaire celle-ci était déjà dans les collections du MAN depuis trois ans (cf. supra); il semble donc logique qu'elle n'y soit pas figurée.

Une hache des collections du musée Dobrée à Nantes (Inv. $\mathrm{n}^{\circ}$ 882.1.327; fig. 11) ressemble très fortement à la pièce $\mathrm{n}^{\circ} 6$ dessinée par Parenteau, les dimensions semblent de plus correspondre relativement bien : 


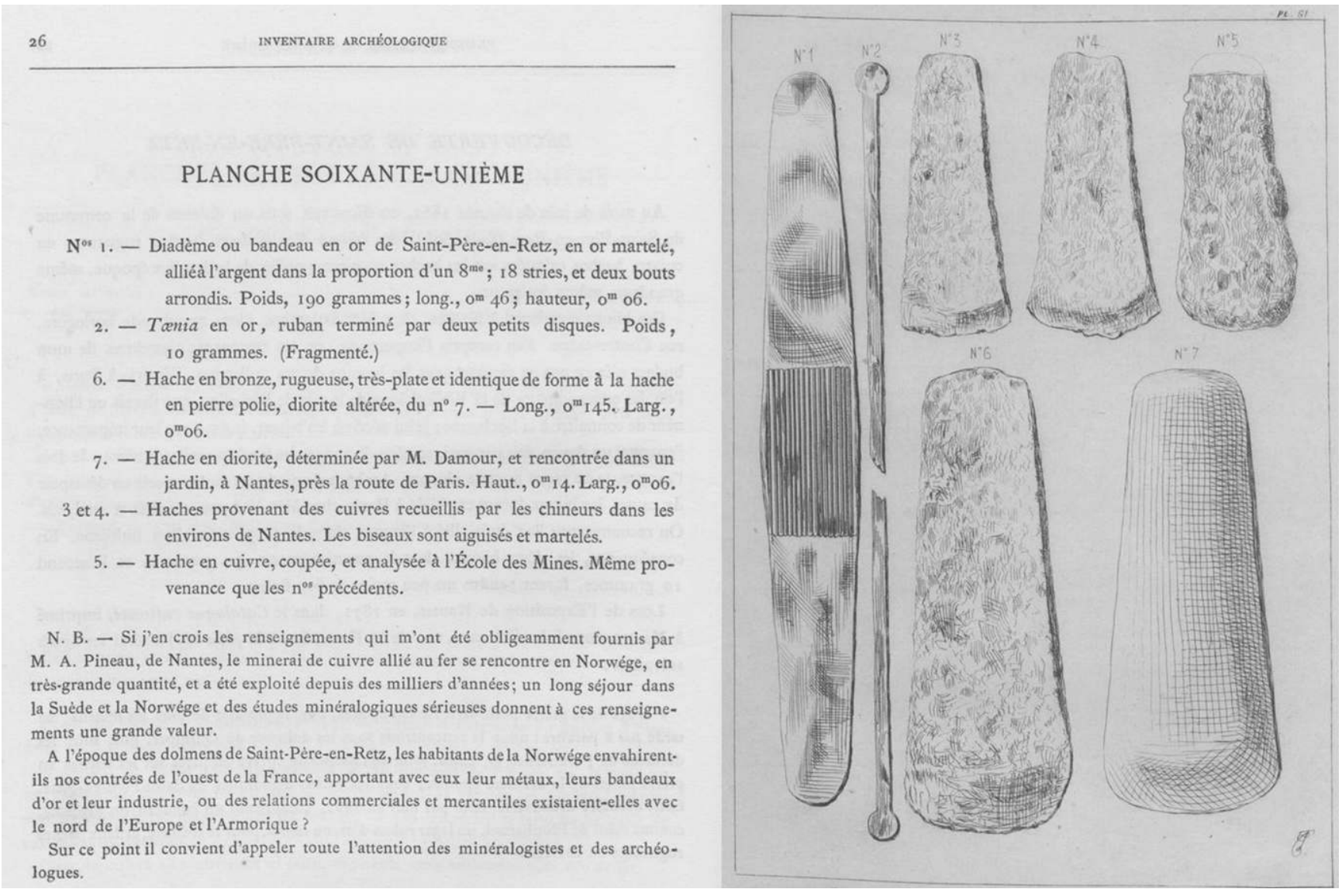

Figure 10 : Planche 61 et sa légende dans l'Inventaire archéologique de F. Parenteau (1878, p. 26 et Pl. 61).

Figure 10: Plate 61 and it's labeling in the "Inventaire archéologique" of F. Parenteau (1878, p. 26 and Pl. 61).

- Parenteau (1878, Pl. 61, no 6) : longueur 14,5 cm; largeur (au tranchant) $6 \mathrm{~cm}$.

- Dobrée (Inv. 882.1.327) : longueur 14,8 cm; largeur (au tranchant) $5,3 \mathrm{~cm}$.

L'inventaire du musée Dobrée indique enfin que cette hache vient précisément de la collection F. Parenteau sous le numéro 107. Outre la similitude des dimensions des haches et leur appartenance à la collection Parenteau, la ressemblance des dessins est suffisamment frappante (notamment la dissymétrie du tranchant) pour que l'on puisse penser qu'il s'agit là du même objet, même si, malheureusement, aucune preuve formelle ne peut venir étayer définitivement cette hypothèse. Enfin, cet objet ressemble assez fortement à la hache de gauche du rapide croquis de F. Parenteau (fig. 7) avec là encore la dissymétrie du tranchant bien marquée. Description de l'objet (fig. 11).

Il s'agit donc d'une hache plate en cuivre / alliage cuivreux (demande d'analyse élémentaire en cours), de forme générale trapézoïdale tout comme la précédente; mais, avec son tranchant moins évasé, elle apparaît comme plus élan- cée. Talon et tranchant sont fins, mais ce dernier, émoussé (avec un léger poli d'usure) et asymétrique présente quatre impacts nets sur le fil. La patine, marron-rouge, est très uniforme et couvre toute la pièce. Les surfaces sont généralement boursouflées et les deux faces offrent de nombreuses traces d'impacts, peut-être dues à un martelage. Le profil de la pièce n'est pas stricto sensu fusiforme mais se rapproche plus d'une forme plano convexe, pouvant peut-être indiquer l'utilisation d'un moule mono-valve sans qu'il soit possible d'être affirmatif sur ce dernier point; néanmoins, aucune bavure de coulée n'est visible sur les flancs, à la différence de la hache de Saint-Germain-en-Laye.

Les deux haches plates du musée Dobrée et de SaintGermain-en-Laye sont donc bien différentes d'un point de vue typologique, la différence de patine semble également indiquer un métal différent pour les deux objets, mais il convient bien sûr de rester très prudent sur les interprétations basées sur la couleur des objets, seuls les résultats des analyses élémentaires pourront confirmer ou infirmer cette première impression visuelle. 


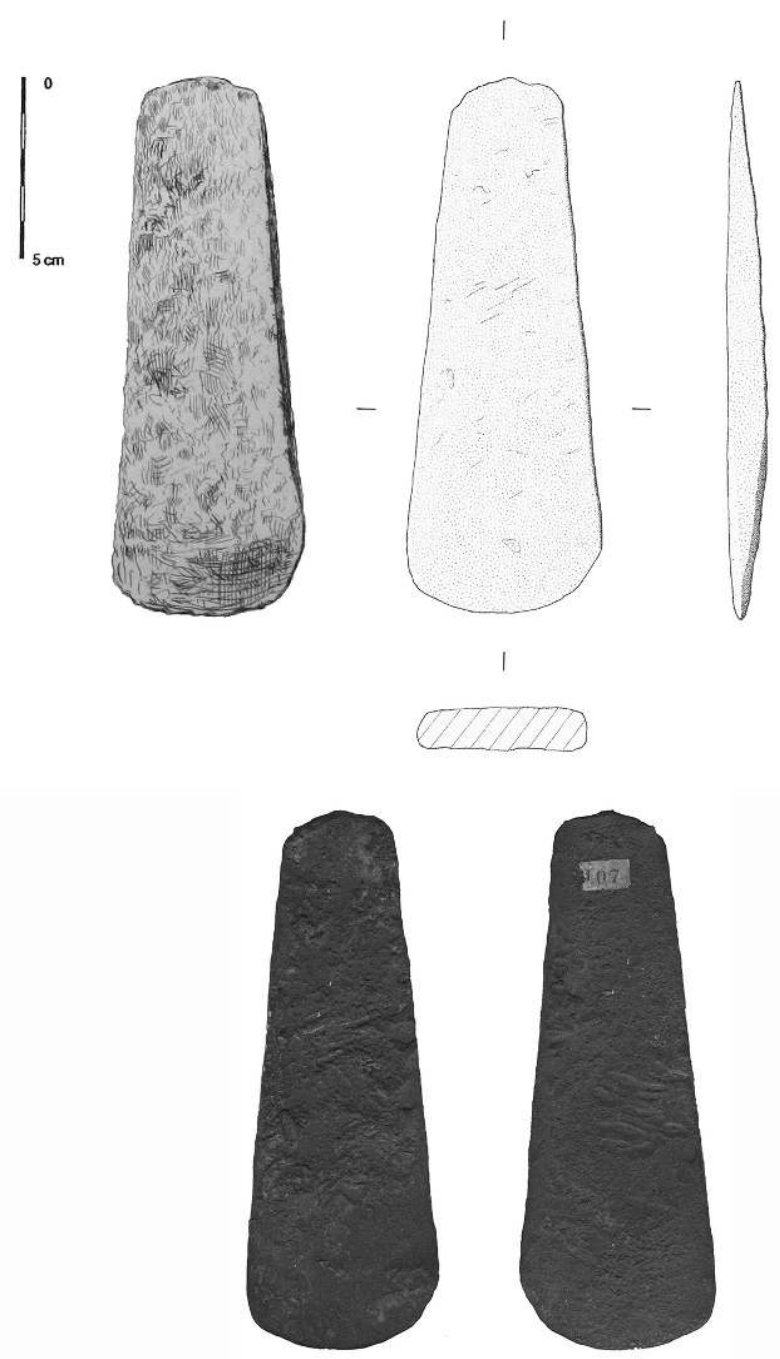

Figure 11 : Hache plate du musée Dobrée (Inv. 882.1.327). D’après Parenteau, 1878, n 6, Pl. 61; dessin H. Gandois; cliché musée Dobrée et sites patrimoniaux - Grand patrimoine de LoireAtlantique).

Figure 11: Flat axe of the Dobrée museum (Inv. 882.13327).

Lieu de conservation : Musée Dobrée, Inv. nº 882.1.327

Longueur $=14,81 \mathrm{~cm}$

Largeur au tranchant $=5,28 \mathrm{~cm}$

Largeur au talon $=3,1 \mathrm{~cm}$

Épaisseur maximale $=1,1 \mathrm{~cm}$

Poids $=402 \mathrm{~g}$

La grande dissemblance entre les deux haches ne plaide clairement pas envers une découverte dans un même ensemble, même si des haches plates trouvées en dépôt peuvent être très proches d'un point de vue typologiques comme par exemple au dépôt de Trentemoult (LoireAtlantique; Baudouin, 1923), mais aussi assez différentes, comme celles du dépôt de La Grassonnière, Le Tablier (Vendée; Baudouin, 1911, p. 38-41; Pautreau, 1979, t. II, fig. 8; Gandois et Barthet, 2013).

\section{Conclusion}

Au travers de l'éclairage que nous livre le fonds Parenteau du musée Dobrée, l'ensemble dit de Saint-Père-en-Retz, abondamment cité dans la littérature, semble donc bien fragile voire même douteux sur beaucoup de points.

Tout d'abord si l'association des haches et des objets en or n'est jamais remise en question quelles que soient les sources (même si les haches sont parfois décrites comme ayant de rebords, Galan et Soutou, 1959, p. 603), il faut bien noter que ces objets sont parvenus chez un orfèvre ou une marchande-horlogère (Parenteau se contredit d'ailleurs sur ce dernier point) à Nantes, et on ne peut donc que se fier aux informations recueillies à ce moment-là.

Ce qui est d'ailleurs désormais certain c'est que ce n'est pas Parenteau qui a fait la découverte, il n'a vu les objets pour la première fois qu'à Nantes, c'est d'ailleurs sans doute à ce moment qu'il s'est porté acquéreur des deux haches, ne pouvant s'offrir les objets en or pour des raisons de budget. Ces derniers ont par la suite été vendus à Paris, le bracelet au moins étant acquis, d'après Chantre (repris par Déchelette) par le célèbre collectionneur Charvet (Chantre, 1875-1876, p. 106-107). Une partie de la collection de ce dernier a été dispersée lors d'une vente aux enchères en 1883. Il n'a malheureusement pas été possible de trouver une quelconque trace du bracelet dans le catalogue de vente (Chevallier et Hoffman, 1883). Peut-être réapparaîtra-t-il à l'occasion d'une succession...

Le lieu de découverte est quant à lui on ne peut plus imprécis, variant de Pornic à Saint-Père-en-Retz via Paimbœuf (communes certes toutes limitrophes), et donc, au final, il faut simplement noter, comme le signalait déjà Parenteau ([b], p. 252) que les quatre objets proviennent du département de Loire-Atlantique et de la rive gauche de la Loire, sans aucune certitude non plus quant à leur découverte sous des " roches druidiques" ou dans un "dolmen ». Cette dernière mention semble, à notre avis, faite $a$ posteriori, à cause de la grande similitude du mobilier du dolmen de Rondossec en Plouharnel (Morbihan).

On peut considérer les dessins des objets comme fiables, Parenteau les ayant bien eu en main avant leur vente et, de manière générale, ses dessins sont de très bonne facture et particulièrement précis.

Si les dessins retrouvés dans les carnets n'apportent que peu d'informations supplémentaires sur les objets en or (la publication de 1878 est suffisamment détaillée), on dispose 
par contre de mesures relativement précises les concernant. Le collier à lamelles d'or découpées mesurait quarante centimètres de longueur une fois déroulé, il a dû être trouvé enroulé à la façon d'un collier car Parenteau en donne le diamètre (treize centimètres, et d'ailleurs la longueur déroulée correspond parfaitement à la circonférence d'un cercle de treize centimètres de diamètre), sa largeur étant de six centimètres. Les stries, de section plano convexe, sont au nombre de dix-huit et couvrent le tiers central du collier, son poids se montant à 190 grammes. Le " torque à palette " ne semble être au final qu'un très fin ruban d'or : trente centimètres de long pour un poids de cinq ou six grammes (selon les versions de Parenteau) et une largeur maximale de cinq millimètres au niveau des extrémités circulaires.

La hache de Saint-Germain-en-Laye (inv. no 22635), malgré la très prometteuse indication de Saint-Père-en-Retz, est très probablement celle de Frossais (Loire-Atlantique), qui a été donnée au musée en 1875 , soit presque vingt-cinq ans après son achat par Parenteau, ce qui peut contribuer à expliquer la confusion. Par contre, une des haches sans provenance de la collection Parenteau du musée Dobrée (inv. no 882.1.327) correspond assez fidèlement au croquis des carnets de Parenteau et, surtout, bien sûr à la représentation donnée dans la publication de 1878, ce qui nous incite donc à la réattribuer à l'ensemble qu'il n'est plus possible d'appeler Saint-Père-en-Retz.

Cette courte étude d'un ensemble, qui semblait pourtant relativement bien connu, est assez révélatrice des confusions et mélanges qui ont pu se produire dans les collections anciennes, et même un objet pourtant dûment identifié par son marquage peut se révéler être d'une provenance différente. Ceci ne peut qu'encourager à utiliser, quand elles existent bien sûr, les vieilles archives des archéologues, antiquaires, érudits locaux : celles-ci contiennent souvent de véritables pépites pour l'étude de ces collections.

\section{Bibliographie}

Baudouin M., I 9 I I - L'âge du Cuivre. Les haches plates en Vendée, Mémoires de la Société préhistorique française, I.

BAudouin M., I923 - « Neuf haches plates et une pointe de javeline, en cuivre pur, trouvées en Loire, près de Trentemoult (Loire-Inférieure) ", Bulletin de la Société préhistorique française, 20 (n $\left.\mathrm{n}^{\circ} 11\right)$, p. 326-329.

Briard J. et L'Helgouach J., I957 - Chalcolithique, Néolithique secondaire, survivances néolithiques à l'âge du Bronze ancien en Armorique, Travaux du laboratoire d'anthropologie et des musées préhistoriques de la faculté des sciences de Rennes.

BRIARD J., I965 - Les dépôts bretons et l'âge du Bronze atlantique, Rennes, travaux du laboratoire d'anthropologie de Rennes.
Chantre E., I 875-I 876 - Études paléo-ethnologiques dans le bassin du Rhône. Âge du Bronze. Recherches sur l'origine de la métallurgie en France, Troisième partie - Statistique, Paris, Librairie Polytechnique de J. Baudry, t. III.

Chevallier P. et Hoffmann H., I883 - Collection Charvet, médailles, antiquités, sceaux-matrices; vente aux enchères publiques, Paris, imprimerie Pillet et Dumoulin.

Déchelette J., I9 Io - Manuel d'archéologie préhistorique, celtique et gallo-romaine, t. II, Archéologie celtique ou protohistorique. Première partie : âge du Bronze (et Appendices), Paris, Librairie Alphonse Picard et fils.

Eluk̀re C., I 977 - "Les premiers ors en France », Bulletin de la Société préhistorique française, études et travaux, $64\left(\mathrm{n}^{\circ} 1\right)$, p. 399-419.

Eluk̀re C., I982 - Les ors préhistoriques, L’âge du bronze en France, t. 2, Paris, Picard.

Galan A. et Soutou A., I959 - « Les boucles d'oreilles rubanées du Midi de la France (début du Hallstatt II) ", Bulletin de la Société préhistorique française, 56, p. 593-603.

Gandois H. et BARThet L., 2013 - " La collection Jolly et la réattribution de quelques haches plates du musée Dobrée ", Bulletin du groupe vendéen d'études préhistoriques, 49, p. $1-13$.

Joussaume R. et Pautreau J.-P., I 990 - La Préhistoire du Poitou, Rennes, Ouest-France Université.

De Mortillet G. et A., I903 - Musée préhistorique, Paris, Schleicher frères.

Musée des Beaux-Arts de Nantes, I 872 - Ville de Nantes : exposition des beaux-arts; archéologie et peinture ancienne, catalogue raisonné, Nantes, Imprimerie Jules Grinsard.

Parenteau F. (a) - Album F. Parenteau 1858-1862, Centre documentaire du musée Dobrée, Nantes, fonds Parenteau, boîte $\mathrm{n}^{\circ} 11$, cote : 997.11.38.

Parenteau F. (b) - Travail préparatoire à l'inventaire. Inventaire archéologique, précédé d'une introduction à l'étude des bijoux, Par F. Parenteau, conservateur du musée départemental d'archéologie de Nantes et de la Loire-Inférieure. Centre documentaire du musée Dobrée, Nantes. Fonds Parenteau, boîte $n^{\circ} 2$, cote : 997.11 .4 .

Parenteau F. (c) - Album F. Parenteau 1858, Centre documentaire du musée Dobrée, Nantes, fonds Parenteau, boîte $\mathrm{n}^{\circ} 4$, cote : 997.11.9.

Parenteau F., i 856 - Catalogue et description des objets d'art du musée archéologique de Nantes et de la Loire-Inférieure, Nantes.

Parenteau F., I 869 - Catalogue du musée départemental d'archéologie de Nantes et de la Loire-Inférieure. Nantes : V. Forest et E. Grimaud, Imprimeurs-Éditeurs.

Parenteau F., I 878 - Inventaire archéologique, Nantes, V. Forest et E. Grimaud, Imprimeurs-Éditeurs.

Pautreau J.-P., I 979 - Le Chalcolithique et l'âge du Bronze en Poitou (Vendée, Deux-Sèvres, Vienne), Poitiers : centre d'archéo- 
logie et d'ethnologie poitevines, musée Sainte-Croix, t. I et t. II.

Riquet R., Guilaine J. et Coffyn A., I963 - " Les campaniformes français (état actuel des recherches) ", Gallia Préhistoire, 6, p. 63-128.
SANtrot M.-H., 200I - «Fortuné Parenteau (1814- 1882) et Pitre de Lisle du Dreneuc (1846-1924), collectionneurs et conservateurs passionnés ", Annales de Bretagne et des Pays de l'Ouest, 118 (n 3), p. 151-242.

Zusammenfassung: Das Fundinventar aus dem „Dolmen von Saint-Père-en-Retz“ (Loire-Atlantique, Pays-de-Loire, France): neue Forschungserkenntnisse - Die Untersuchung der Flachbeile aus den Sammlungen des Museums Dobrée in Nantes und derjenigen des Musée d'archéologie nationale (MAN) in Saint-Germain-en-Laye haben es gestattet, ein Beil wiederaufzufinden, dass die Bezeichnung "Saint-Père-en-Retz, Loire-Inférieure" trägt. Das archäologische Fundmaterial (zwei Goldobjekte sowie zwei Flachbeile aus Kupfer bzw. einer Kupferlegierung), das in einem „Dolmen“ in dieser Gemeinde gefunden worden war, gilt allgemein als typisch für die Glockenbecherkultur und wird entsprechend häufig in der Fachliteratur zitiert. Die Funde sind von F. Parenteau, dem ersten Konservator des Musée départemental d'Archéologie des Departments Loire-Atlantique, geborgen worden. Die Überprüfung der sehr reichen Dokumentation zu diesen Funden hat es ermöglicht, einige bisher unveröffentlichte Dokumente zu sichten, die neue Informationen zu diesem Fundensemble liefern. Es hat sich dabei herausgestellt, dass diese Funde nicht von Parenteau selbst entdeckt wurden wie er a posteriori 1872 schreibt (Musée des Beaux-Arts von Nantes, 1872, p. 36), sondern aus zweiter Hand von einem Goldschmied in Nantes erworben wurden. Die Aufzeichnungen von Parenteau erlauben es ferner anzunehmen, dass die Lokalisierung der Funde (Saint-Père-en-Retz) und ihr archäologischer Kontext („Dolmen“) wenig gesichert sind, ebenso wie die Umstände ihrer Entdeckung. In der Folge kommt es in einigen Publikationen zu weiteren, sehr fantasievollen Ausschmückungen der Fundgeschichte. Einige bisher unveröffentlichte Zeichnungen aus dem Konvolut der Aufzeichnungen Parenteaus vervollständigen die bisher bekannte Dokumentation dieser Funde, ziehen jedoch die genannte Herkunft des im MAN verwahrten Flachbeiles in Zweifel und erlauben es, eines der im Musée Dobrée verwahrten Beile dem Fundensemble hinzuzufügen. Zusammenfassend kann gefolgert werden, dass die neuen Erkenntnisse das bekannte und sehr umfangreich zitierte Fundensemble hinsichtlich seiner Lokalisierung sowie seines Fundkontextes als ausgesprochen zweifelhaft erscheinen lassen. Es zeigt sich, dass das Studium der alten Archivunterlagen aus dem 19. Jahrhundert sich häufig als eine unverzichtbare Hilfe bei der Analyse und Überprüfung alter Sammlungsbestände erweist.

Resumen: El material del “dolmen de Saint-Père-en-Retz” (Loire-Atlantique): nuevos elementos de reflexión-El estudio de hachas planas de las colecciones del museo Dobrée en Nantes y del Museo Arqueológico Nacional (MAN) en Saint-Germain-en-Laye permitió encontrar un hacha marcada por la inscripción "Saint-Père-en-Retz, Loire-Inférieure". El material que se habría encontrado en un "dolmen" de este municipio (dos artefactos de oro y dos hachas planas de cobrelaleación de cobre) es a menudo visto como emblemático de la cultura del campaniforme y citado ampliamente en la literatura. Los artefactos fueron recogidos por F. Parenteau, primer conservador del museo departamental de arqueología de Loire-Atlantique. La consultación de la rica documentación de este hombre erudito ha ayudado para recuperar algunos documentos inéditos que aportan nuevos elementos a propósito de este conjunto. Demuestra, entre otros, que los objetos no fueron descubiertos por Parenteau si mismo como escribió retrospectivamente en 1872 (Museo de Bellas Artes de Nantes, 1872, p. 36) pero que fueron comprados de segunda mano a un orfebre de Nantes. Las notas de Parenteau permiten darse cuenta de que la ubicación (Saint-Père-en-Retz) y el contexto ("Dolmen") del depósito no son fiables y, esto, en el momento mismo del descubrimiento. Luego, varias publicaciones han añadido materiales, a veces de poca credibilidad. Dibujos inéditos del fondo Parenteau completan la documentación de este conjunto, pero cuestionan también fuertemente el origen de la hacha plana del MAN, mientras que, al mismo tiempo, permiten adjuntar una de las hachas del museo Dobrée a este conjunto. Al final, los nuevos elementos reunidos indican que el conjunto famoso y mencionado con profusión es muy dudoso, tanto desde el punto de vista de la ubicación como él del contexto, y que la consultación de los archivos del siglo XIX aparece a menudo como ayuda inestimable para el estudio de las colecciones antiguas.

Schlüsselwörter: Flachbeil, Parenteau, Dolmen, Goldfunde, Gargantilla, Chalkolithikum, Frühe Bronzezeit, Saint-Père-en-Retz, Dobrée-Museum.

Palabras clave: Hacha plana Parenteau, dolmen, material de oro, gargantilla, Calcolitico, Edad del Cobre, Edad del Bronce Antiguo, Saint-Père-enRetz, museo Dobrée. 